\title{
APLICACIÓN DE UN MODELO CUANTITATIVO OBJETIVO PARA LA MEDICIÓN DE LA COMPETITIVIDAD DE LOS DESTINOS TURÍSTICOS ${ }^{1}$
}

\author{
José Miguel Rodríguez-Antón \\ Luis Rubio-Andrada \\ María del Mar Alonso-Almeida \\ María Soledad Celemín-Pedroche \\ Universidad Autónoma de Madrid
}

\section{RESUMEN}

El objetivo del presente trabajo consiste en proponer un modelo cuantitativo objetivo de competitividad de los países como destinos turísticos. Este modelo, basado en el propuesto por el World Economic Forum, está compuesto exclusivamente por indicadores extraídos de bases de datos y de estadísticas elaboradas por instituciones públicas o privadas. Para determinar los pesos que cada uno de los pilares y variables que lo integran, se distribuyó un cuestionario a una muestra formada por 186 profesionales del sector turismo, especialistas del sector turismo y profesores universitarios, que dio lugar a un modelo lineal de competitividad turística, el cual fue aplicado a los 28 países que integran la Unión Europea, resultando que Alemania es el país con mayor competitividad turística, seguido por Italia, Reino Unido, Francia y España.

Palabras clave: Competitividad turística, turismo, destinos turísticos, modelo de competitividad, indicadores cuantitativos objetivos.

Recibido: 22 de abril de 2015

Devuelto para su revisión: 2 de julio de 2015

Aceptado: 24 de septiembre de 2015

Facultad de Ciencias Económicas y Empresariales. Universidad Autónoma de Madrid. 28049 Cantoblanco. MADRID (España). E-mail: josem.rodriguez@uam.es, luis.rubio@uam.es, mar.alonso@uam.es, marisol.celemin@uam.es

1 Este artículo se enmarca en el Proyecto de Investigación competitivo titulado «Análisis de la competitividad de España como destino turístico. Propuesta de un modelo de medición de intangibles turísticos», que ha sido financiado por la Universidad Autónoma de Madrid (Ref. CEMU 2013-15) y que ha sido desarrollando por un Equipo de Investigación del que forman parte los autores del mismo. El texto íntegro de dicho Proyecto puede verse en Rodríguez Antón, dir. (2015). 


\title{
Application of a quantitative-objective model for measuring the competitiveness of tourist destinations
}

\begin{abstract}
The aim of this paper is to propose a quantitative objective model for measuring the competitiveness of countries as tourist destinations. This model, based on the one proposed by the World Economic Forum, is exclusively composed of indicators which were drawn from databases and statistics made by public or private institutions. In order to determine the weights of the pillars and variables that comprise the model, a questionnaire was distributed to a sample of 186 professionals in the tourism sector, tourism industry experts and academics, resulting in a linear model of tourism competitiveness. Furthermore, this model was applied to the 28 countries comprising the European Union, with the result that Germany is the country with the highest tourism competitiveness, followed by Italy, UK, France and Spain.
\end{abstract}

Keywords: Tourist competitiveness, tourism, tourist destinations, competitive model, quantitative objective indicators.

\section{INTRODUCCIÓN}

No es necesario incidir en la importancia que el turismo tiene para la mayor parte de las economías y, especialmente, para la española. Buena parte de la reactivación económica que estamos experimentando en estos últimos años ha sido debida a este sector. Además, en el último informe publicado por el World Economic Forum (2015), España se situaba en la primera posición del ranking mundial por nivel de competitividad turística. Este hecho, nunca antes logrado, ha supuesto que nos pongamos, por primera vez desde que se confecciona este ranking, por delante de nuestros más importantes competidores en el ámbito del turismo receptivo.

Sin embargo, no basta con llegar a esta privilegiada posición. Es necesario mantenernos en ella durante el periodo más amplio posible. Al igual que, a nivel micro, se pretende que las ventajas competitivas de una empresa sean sostenibles en el tiempo, la competitividad turística, a nivel macro, también debe buscar consolidar este nivel en el tiempo. Para ello, es necesario que nuestro país cuide de forma continua aquellos factores que integran o determinan nuestra competitividad como destino turístico.

En consecuencia, se hace imprescindible analizar en profundidad los factores que van a determinar la competitividad de un destino turístico para, posteriormente, medir su nivel o grado de consecución y, a continuación, poner un énfasis especial en aquellos aspectos que sean más influyentes en la competitividad turística. Para ello, en el presente trabajo se va a proponer la creación de un modelo cuantitativo objetivo de competitividad de los destinos turísticos (MCOCDT) que facilite este proceso.

En el ámbito específico del turismo, un buen número de autores como De Keyser y Vanhove (1994, p. 19), Hassan (2000, p. 239), D’Hauteserre (2000, p. 23) o Hong (2008, p. 6), partiendo de las aportaciones clásicas de Ricardo (1817) y Porter (1985, 1990), han 
propuesto definiciones relacionadas con la competitividad turística. Desde nuestro punto de vista, la competitividad de un destino es «la capacidad que posee un área geográfica -dotada de unas características específicas atrayentes- de competir con otras áreas geográficas debido a que posee unos recursos extraordinarios o a que los gestiona de una manera eficiente».

La competitividad de los destinos turísticos ha sido objeto de un amplio conjunto de estudios entre los que merecen destacarse los de Crouch y Ritchie (1999), D'Hauteserre (2000), Saveriades (2000), Weaver (2000), Costa (2001), Kim et al. (2001), Orta (2005) Jang y Feng (2006), Claver, Molina y Pereira (2007), Gomezelj y Mihalic (2008), González y Mendieta (2009), Cracolici y Nijkamp (2009), Hong (2009) y Perles-Ribes, RamónRodríguez y Sevilla-Jiménez (2014). Algunos de estos autores han llegado a proponer modelos de competitividad turística que, en algunos casos, son meramente teóricos o conceptuales y pretenden analizar los factores y circunstancias que pueden determinar o influir en la competitividad de los destinos, pero que en otros son empíricos y buscan llegar a medir, de manera cuantitativa, la competitividad que poseen dichos destinos.

De entre los modelos de competitividad turística existentes, los más referenciados por la literatura son los modelos de Crouch y Ritchie (Crouch y Ritchie, 1999; Ritchie y Crouch, 2000; Ritchie y Crouch, 2003), el modelo de Dwyer y Kim (Dwyer y Kim, 2003 y Dwyer, Knezevic, Mihalic y Koman, 2014), el Modelo de Hong $(2008,2009)$ y el Modelo del World Economic Forum (WEF, 2007, 2009, 2011, 2013, 2015). Otros modelos, como el Monitor de Competitividad, creado en 2001 por el World Travel and Tourism Council -del que parten el modelo de Mazanec, Wöber y Zins (2007) y el de Jiménez y Aquino (2012)-, el modelo de De Keyser y Vanhove (1994), el de Kozak y Rimmington (1999),el de Monfort (1999) y el de Garau (2006), entre otros muchos, han tenido una orientación eminentemente práctica y enfocada a la medición de la competitividad de áreas, países o grandes zonas geográficas.

\section{PROPUESTA DE CREACIÓN DE UN MODELO CUANTITATIVO OBJETIVO DE COMPETITIVIDAD DE LOS DESTINOS TURÍSTICOS (MCOCDT)}

Si realizamos una comparación de los cuatro modelos principales anteriormente citados, -modelos de Crouch y Ritchie, de Dwyer y Kim, de Hong y del WEF- podemos comprobar que todos ellos realizan un esfuerzo por proponer un reducido número de grandes factores de competitividad, aunque los descomponen en un número muy variable de atributos y de indicadores. De hecho, el modelo de Crouch y Ritchie no llega a definir indicadores, manteniéndose en un ámbito puramente teórico. Un estudio comparado de estos modelos puede verse en Rodríguez-Antón, dir. (2015).

De estos modelos, se ha seleccionado el del WEF -en concreto la edición de 2013, puesto que a partir de ella se ha realizado el estudio base de esta comunicación-, porque es el que mejor se adecua para servir de base a nuestra propuesta de creación de un modelo cuantitativo objetivo de competitividad turística, debido a varios motivos:

- Este modelo posee una dilatada trayectoria de aplicación empírica a la inmensa mayoría de los países del mundo, comenzando a aplicarse en 2007.

- Se publica con regularidad, en concreto, bienalmente. 
- La última versión del citado modelo es muy reciente -se realizó en mayo de 2015-.

- Se aplica a un importante número de países (en la versión de 2013 se aplicó a 140 países y en la de 2015 a 141).

- Este modelo ha sido abundantemente citado en la literatura científica y ha sido utilizado para el desarrollo de múltiples trabajos de investigación relacionados con la competitividad turística.

- Aunque parece ser más simple que otros modelos de competitividad, ya que cuenta con un reducido número de grandes factores de competitividad -tres en la versión de 2013 y cuatro en la de 2015-, a través de ellos se recogen la mayor parte de los atributos de los que constan los otros modelos.

- Este modelo propone una serie de indicadores para cada uno de los atributos o pilares definidos, ofreciendo un listado de 79 indicadores de competitividad en la versión de 2013, que suponen una buena aproximación a la medida de la competitividad de los destinos turísticos por ser bastante concretos, pudiendo ser cuantificados.

Sin embargo, todos estos aspectos positivos se ven relativizados, por el hecho de que buena parte de estos indicadores definidos por el WEF no pueden ser medidos de manera objetiva, sino que lo son sobre la base de las opiniones de expertos. No se trata de volver a aflorar las reflexiones que se realizaron a mediados del siglo pasado relacionadas con un posible enfrentamiento entre los paradigmas cuantitativo y cualitativo, pues existe una abundante bibliografía al respecto. Autores como Campbell y Fiske (1959), Campbell (1970), Campbell y Overman (1988), Cook y Reichardt (1986) y Hernández (1998), entre otros, han puesto de relieve las ventajas y los inconvenientes de la utilización de ambos paradigmas metodológicos en la investigación. Sin embargo, el conocimiento científico debe procurar ir más allá de la subjetividad buscando la precisión y el rigor de una manera fiable. De hecho, el paradigma cuantitativo se utiliza en mayor medida que el cualitativo a la hora de contrastar modelos. Esta opinión se ha visto refrendada muy recientemente por Dwyer et al. (2014), que defienden la reducción, en la medida de lo posible, de medidas blandas basadas en opiniones de expertos, aduciendo que la opinión de una persona sobre un hecho no va a tener el valor de un dato objetivo concreto relacionado con dicho hecho. Por ello, proponemos, como metodología de aproximación a la creación de un modelo cuantitativo objetivo de competitividad de los destinos turísticos, la búsqueda y posterior utilización de indicadores objetivos obtenidos a través de bases de datos y fuentes estadísticas oficiales contrastadas.

El modelo base seleccionado -el del WEF (2013) - está integrado por 3 subgrupos de competitividad, 14 pilares y 79 indicadores. El primer subgrupo, denominado Marco regulatorio turístico, cuenta con cinco pilares: Política y regulación, Sostenibilidad medioambiental, Seguridad, Salud e higiene y Priorización de la industria turística, que engloban un total de 29 indicadores. El segundo subgrupo, relativo al Entorno empresarial e infraestructuras turísticas, posee otros cinco pilares: Infraestructuras de transporte aéreo, Infraestructuras de transporte por tierra, Infraestructura turística, Infraestructuras de telecomunicaciones y competitividad en precio, que suponen 27 indicadores. Por último, el subgrupo Recursos humanos, culturales y recursos naturales turísticos, posee otros cuatro pilares: Recursos Humanos, Afinidad a la industria turística, Recursos naturales y Recursos culturales, que se descomponen en 23 indicadores. 
El índice integrado en este modelo se aplicó a 140 países y está basado en datos procedentes tanto de una encuesta de opinión remitida a expertos internacionales como de datos cuantitativos procedentes de fuentes externas a esta organización. Tras el análisis de los 79 indicadores propuestos por este modelo, se ha comprobado que nada menos que 31 eran de tipo blando, es decir, estaban basados en opiniones ofrecidas por expertos conseguidas, en concreto, a través de la Encuesta de Opinión Ejecutiva elaborada por el propio World Economic Forum.

Una vez identificados estos indicadores blandos, se han buscado otros, de tipo duro u objetivos, en fuentes estadísticas o en bases de datos de ámbito internacional que midiesen la misma variable y que pudiesen sustituir a los originales. Este proceso no siempre ha sido posible por no existir, en algunos casos, ninguna fuente estadística que recogiese esa variable a medir. Cuando se ha producido esta circunstancia, se ha optado por buscar otra variable $-\mathrm{y}$, por ende, otro indicador- alternativa que pudiese recoger de manera aproximada el elemento de competitividad que originariamente quería medir el WEF.

En la Tabla 1 se recogen el número de variables que define el modelo del WEF (2013), el número de indicadores de dichas variables que se han podido encontrar en las bases de datos y estadísticas accesibles y los nuevos indicadores que se han incluido -tan sólo dos- para completar dos pilares de los que no se había podido encontrar indicadores cuantitativos objetivos directos sustitutos de las variables que habían sido medidas por el WEF de forma blanda. De forma resumida, de las 79 variables consideradas por el WEF (2013), se ha pasado a 60 variables cuantitativas objetivas directas más dos variables cuantitativas objetivas nuevas, lo cual supone, en cierta forma, una simplificación del modelo original.

\section{Tabla 1}

NÚMERO DE INDICADORES DEL WEF (2013) E INDICADORES CUANTITATIVOS OBJETIVOS ENCONTRADOS Y PROPUESTOS

\begin{tabular}{|c|c|c|c|c|}
\hline PILAR & NOMBRE & \begin{tabular}{|c} 
VARIABLES \\
DEL WEF \\
$(2013)$
\end{tabular} & $\begin{array}{c}\text { INDICADORES } \\
\text { CUANTITATIVOS } \\
\text { OBJETIVOS } \\
\text { ENCONTRADOS }\end{array}$ & $\begin{array}{c}\text { NUEVOS } \\
\text { INDICADORES } \\
\text { CUANTITATIVOS } \\
\text { OBJETIVOS } \\
\text { PROPUESTOS } \\
\end{array}$ \\
\hline 1 & POLITICA YREGULACIOON & 9 & 7 & 1 \\
\hline 2 & SOSTENIBILIDAD AMBIENTAL & 7 & 5 & \\
\hline 3 & SEGURIDAD & 4 & 4 & \\
\hline 4 & SALUD E HIGIENE & 4 & 4 & \\
\hline 5 & PRIORIZACIÓN DE LOS VIAJES Y EL TURISMO & 5 & 2 & 1 \\
\hline 6 & INFRAESTRUCTURAS DEL TRANSPORTE AÉREO & 7 & 2 & \\
\hline 7 & INFRAESTRUCTURAS DEL TRANSPORTE TERRESTRE & 5 & 4 & \\
\hline 8 & INFRAESTRUCTURAS TURISTIICAS & 3 & 3 & \\
\hline 9 & INFRAESTRUCTURAS DE TELECOMUNICACIONES & 7 & 6 & \\
\hline 10 & COMPEEITIVIDAD DE PRECIOS EN LA INDUSTRIA DEVIAJES Y TURISMO & 5 & 4 & \\
\hline 11 & RECURSOS HUMANOS & 10 & 9 & \\
\hline 12 & AFINIDAD POR LOS VIAJES Y EL TURISMO & 4 & 1 & \\
\hline 13 & RECURSOS NATURALES & 5 & 5 & \\
\hline 14 & RECURSOS CULTURALES & 4 & 4 & \\
\hline
\end{tabular}

Fuente: Rodríguez-Antón, dir. (2015). 
A modo de ejemplo, no se han incluido variables como Impacto en los negocios de las normas existentes relacionadas con las inversiones directas extranjeras, Comprensión de los datos mensuales de la industria turística, Calidad de las infraestructuras del transporte aéreo o Actitud de la población local hacia los turistas extranjeros, todas ellas de tipo blando y muy difícilmente evaluables de manera cuantitativa objetiva.

Como resumen, es preciso hacer notar que si bien de la práctica totalidad de pilares que constituyen el modelo se han podido encontrar casi tantos indicadores cuantitativos objetivos como número de variables poseía el modelo original, en el caso de los pilares 5. Priorización de los viajes y el turismo, 6. Infraestructuras del transporte aéreo y 12. Afinidad por los viajes y el turismo, esto no ha sido posible debido a que, en algún caso, las variables definidas en el modelo de referencia no tenían indicadores de acceso libre y a que, en otros, las variables estaban definidas, en dicho modelo, sobre la base de opiniones del propio WEF y no sobre datos reales.

Una vez que se han seleccionado todos aquellos indicadores de tipo duro que estaban disponibles en fuentes estadísticas o en bases de datos de libre acceso y que eran capaces de medir, de forma cuantitativa objetiva, los distintos pilares que configuran el modelo del WEF (2013), se configuró un instrumento de medida que sirvió para recoger las opiniones de expertos relativas a los pesos relativos que debían tener tanto cada uno de los catorce pilares del modelo como las variables seleccionadas de cada uno de ellos.

Para ello, se creó un cuestionario que fue testado en diciembre de 2014 por cuatro expertos, tres profesores universitarios y un profesional del sector. A partir de estas opiniones, se confeccionó un cuestionario definitivo que fue colocado en la plataforma Survey Monkey desde el día 12 de enero de 2015 hasta el 20 de febrero de 2015. Los encuestados fueron seleccionados a partir de una muestra de conveniencia constituida por tres colectivos de relevancia en el sector turismo: Profesionales del sector turismo, profesores universitarios de turismo y especialistas en turismo. Todos ellos fueron contactados a través del envío de correos electrónicos personalizados en los que se les solicitaba que dieran su opinión sobre los pesos que debían tener cada pilar y cada variable a la hora de crear un modelo cuantitativo de medición de la competitividad de los destinos turísticos.

Todas las preguntas eran cerradas, en una escala Likert de 11 puntos -de 0 a $10-$ y, además de su opinión sobre la importancia de cada pilar y sus variables, se les pidieron una serie de datos personales que permitiesen caracterizar la muestra lograda (profesión, género, edad y años de experiencia en el sector turismo).

En total, se lograron 186 respuestas válidas de los tres colectivos con la siguiente distribución:

a) Profesionales del sector turismo: $24,2 \%$

b) Profesores universitarios de turismo: $55,4 \%$

c) Especialistas en turismo: $8,1 \%$

El resto de encuestados marcaron otras opciones residuales o varias de ellas a la vez.

Por otro lado, el 58,2 por ciento de las personas que respondieron fueron hombres y el 41,8 por ciento mujeres, el mayor porcentaje de personas que respondieron -el 39,1 por 
ciento- tenía una edad comprendida entre los 41 y los 50 años, y la mayor parte -el 24,4 por ciento- contaba con una experiencia en el sector superior a los 20 años.

Una vez que se obtuvieron las opiniones de los encuestados, se realizó el tratamiento de datos correspondiente. Para ello, en primer lugar se trataron las respuestas obtenidas para cada uno de los catorce pilares existentes. Se trataba de conocer la importancia que estos colectivos daban a cada uno de estos pilares a la hora de definir y medir la competitividad de los destinos turísticos. Ello se consiguió sumando las puntuaciones que los 186 encuestados habían dado a los catorce pilares y se calculó el tanto por mil que cada uno de esos pilares suponía en la valoración global otorgada. De esa forma, la suma de las ponderaciones logradas por los catorce pilares salía mil y, por tanto, la ponderación de cada pilar se daba en tantos por mil.

A continuación, se distribuyó, para cada pilar, la ponderación obtenida en el paso anterior entre las variables que lo configuraban. De esa manera, cada pilar seguía manteniendo la importancia o el peso calculado anteriormente, y se calcularon las ponderaciones de cada variable que integraba cada pilar en su propio pilar. De esa forma, la suma de las ponderaciones de todas las variables de un pilar daba como resultado la ponderación calculada para dicho pilar.

Por último, el cálculo de la competitividad de cada destino turístico se realizó a través del sumatorio de cada ponderación de todas las variables integradas en todos los pilares multiplicado por los valores reales de dichas variables. Por tanto, el indicador de competitividad cuantitativo objetivo logrado se configura como una ecuación lineal del tipo:

$$
I C P_{i}=\sum_{j=1}^{\leq 62} I_{i j} \times w_{j}
$$

siendo $\mathrm{ICP}_{\mathrm{i}}$ el índice de competitividad del país i

$I_{i j}$ el indicador j para el país i, cumpliéndose siempre que $0 \leq I_{i j} \leq 1$

$W_{j}$ la ponderación del indicador j, común para todos los países resultando, por tanto, que el modelo que lo sustenta también es de carácter lineal.

Aunque el WEF (2013) calcula la competitividad para un total de 140 países, en el presente trabajo se decidió circunscribir la aplicación práctica del modelo a los veintiocho países que integran actualmente la Unión Europea. Esta elección viene justificada por varios motivos, pero el de mayor peso se basa en que la mayor parte de nuestros principales competidores se encuentran en este espacio europeo.

Es necesario hacer notar que cuando algún valor de alguna variable no se ha podido obtener por no estar disponible en las fuentes estadísticas o bases de datos para un país concreto, el peso de esta variable en el pilar correspondiente se ha distribuido entre las que sí había información, haciéndose una reponderación, de tal forma que su indisponibildiad no redujese el peso de dicho pilar en la medida de competitividad creada.

Dado que las variables utilizadas son muy heterogéneas, tanto por la amplia gama de unidades utilizadas para su medida (euros, porcentajes, personas, ratios, etc.) como por el valor logrado (unidades, miles, millones, etc.), se ha considerado necesario reescalar 
dichos valores de dos formas. Si una variable influía positivamente en la competitividad de un destino turístico, se ha reescalado para que el mayor -mejor-valor tuviese un valor de 1, bajando de forma proporcional a cada valor obtenido (un índice simple cuyo denominador era el valor máximo del grupo de países considerados). Por contra, cuando la variable influía negativamente en esta competitividad, se ha reescalado para que el valor 1 fuese también el mejor valor pero, en este caso, el valor menos negativo para la competitividad (el inverso de un índice simple cuyo denominador era el valor mínimo del grupo de países considerados) ${ }^{2}$.

\section{RESULTADOS}

De los catorce pilares de los que consta el modelo, el que más influye en la competitividad de un destino turístico es, en opinión de los encuestados, el constituido por las Infraestructuras del transporte aéreo, logrando una valoración media de 8,694 en una escala Likert de 0 a 10 puntos. A continuación se situarían las Infraestructuras turísticas con una valoración de 8,632 y la Seguridad y protección con una valoración de 8,608. En cuarto lugar se situarían los Recursos culturales con 8,408, seguidos por la Salud e higiene con 8,333 y los Recursos naturales con 8,315. Los pilares peor considerados por los expertos fueron la Prioridad del turismo y de los viajes para el destino, con 7,503 y la Sostenibilidad ambiental con 7,581 (ver Tabla 2).

Tabla 2

\section{VALORACIÓN DE LOS PILARES QUE CONFIGURAN EL MODELO CUANTITATIVO OBJETIVO DE COMPETITIVIDAD DE LOS DESTINOS TURÍSTICOS (MCOCDT)}

\begin{tabular}{|l|c|c|}
\hline \multicolumn{1}{|c|}{ PILARES DE COM PETITIVIDAD } & N & Media \\
\hline P1. POLÍTICA Y REGULACIÓN & 186 & 7,710 \\
\hline P2. SOSTENIBILIDAD AMBIENTAL. & 186 & 7,581 \\
\hline P3. SEGURIDAD Y PROTECCIÓN & 186 & 8,608 \\
\hline P4. SALUD E HIGIENE & 186 & 8,333 \\
\hline P5. PRIORIDAD DEL TURISMO Y DEL SECTOR DE VIAJES PARA EL DESTINO & 186 & 7,503 \\
\hline P6. INFRAESTRUCTURAS DEL TRANSPORTE AÉREO & 186 & 8,694 \\
\hline P7. INFRAESTUCTURAS DEL TRANSPORTE TERRESTRE & 186 & 8,306 \\
\hline P8. INFRAESTRUCTURAS TURÍSTICAS & 186 & 8,632 \\
\hline P9. INFRAESTUCTURAS DE TELECOMUNICACIONES (TIC) & 186 & 7,935 \\
\hline P10. COMPETITIVIDAD DE LOS PRECIOS DE LA INDUSTRIA TURÍSTICA & 186 & 8,048 \\
\hline P11. RECURSOS HUMANOS & 186 & 8,071 \\
\hline P12. GRADO DE APERTURA A LOS VIAJES Y AL TURISMO & 186 & 7,780 \\
\hline P13. RECURSOS NATURALES & 186 & 8,315 \\
\hline P14. RECURSOS CULTURALES & 186 & 8,408 \\
\hline
\end{tabular}

Fuente: Rodríguez-Antón, dir. (2015).

2 Un análisis factorial exploratorio de este modelo puede verse en Rodríguez Antón y Rubio (2016). 
A continuación, se calcularon las ponderaciones de cada una de las 62 variables en las que se descomponen estos 14 pilares que configuran el MCOCDT y se multiplicaron por los valores cuantitativos de dichas variables, lo que ha permitido calcular la competitividad de los 28 países que integran la Unión Europea.

Como puede observarse en la Tabla 3, el país que ha logrado obtener el mayor índice de competitividad es Alemania, consiguiendo un total de 631,589 puntos sobre un máximo posible de 1.000 puntos. A continuación se ha situado Italia, seguido por el Reino Unido, Francia y España.

Tabla 3

RANKING DE COMPETITIVIDAD

DE LOS PAÍSES DE LA UE SEGÚN EL MCOCDT

\begin{tabular}{|l|c|}
\hline PAIS & ÍNDICE \\
\hline ALEMANIA & 631,589 \\
\hline ITALIA & 626,846 \\
\hline R.UNIDO & 603,351 \\
\hline FRANCIA & 578,918 \\
\hline ESPAÑA & 550,431 \\
\hline LUXEMBURG O & 543,005 \\
\hline BELGICA & 487,519 \\
\hline DINAMARCA & 484,853 \\
\hline AUSTRIA & 484,252 \\
\hline BULGARIA & 477,704 \\
\hline HOLANDA & 466,542 \\
\hline GRECIA & 455,396 \\
\hline PORTUGAL & 448,544 \\
\hline CROACIA & 444,179 \\
\hline REP.CHECA & 441,172 \\
\hline SUECIA & 431,636 \\
\hline MALTA & 430,337 \\
\hline IRLANDA & 421,258 \\
\hline POLONIA & 415,925 \\
\hline FINLANDIA & 415,245 \\
\hline CHIPRE & 414,769 \\
\hline ESLOVAQUIA & 410,894 \\
\hline HUNG RIA & 407,185 \\
\hline ESTONIA & 396,258 \\
\hline ESLOVENIA & 392,153 \\
\hline LETONIA & 390,965 \\
\hline LITUANIA & 388,752 \\
\hline RUMANIA & 381,019 \\
\hline
\end{tabular}

Fuente: Rodríguez-Antón, dir. (2015). 
Si descomponemos la competitividad de estos destinos turísticos por cada uno de los catorce pilares considerados, podemos comprobar que mientras para los dos pilares considerados más influyentes en la competitividad de un destino -las Infraestructuras del transporte aéreo y las Infraestructuras turísticas- los grandes países receptores de turismo son los que ocupan las primeras posiciones -Reino Unido, Alemania y Francia en el primer caso e Italia, Alemania y España, en el segundo-, para el tercer pilar por importancia según los expertos consultados, la Seguridad y protección, los primeros puestos son ocupados por Bulgaria, Francia y Rumanía.

España se sitúa en primera posición de la Unión Europea atendiendo al Pilar 2. Sostenibilidad ambiental, en segunda posición por el Pilar 14. Recursos culturales y en tercera por los Pilares 5. Prioridad del turismo y del sector de viajes para el destino y 8 . Infraestructuras turísticas. Sin embargo, no estamos muy bien posicionados en el Pilar 9. Infraestructuras de telecomunicaciones ni en el Pilar 12. Grado de apertura a los viajes y al turismo ni en el Pilar 4. Salud e higiene ni, lo que es bastante más grave, en el Pilar 11. Recursos humanos.

Tras la aplicación del MCOCDT y la posterior elaboración del ranking de países de la Unión Europea por su nivel de competitividad, se ha efectuado un análisis comparado con los resultados logrados para esos mismos países por el modelo de referencia -el WEF(2013)-, detectándose la existencia de una cierta correlación entre los valores obtenidos por ambos modelos, la cual, medida a través del coeficiente de correlación de Pearson, se eleva a 0,646 , lo cual indica que ambos modelos tienen cierta relación, pero no son idénticos, lo cual era de prever al partir de los mismos pilares y variables, pero sustituyendo todos los indicadores blandos empleados por el WEF por indicadores duros. Además, se ha detectado que el modelo propuesto recoge un mayor número de matices diferenciales pues el coeficiente de variación obtenido para el nuevo modelo propuesto se sitúa en 0,157 , en tanto que el obtenido para el modelo de referencia se queda en 0,075 .

Si comparamos las posiciones que ocupan los veintiocho países de la UE según nuestro ranking o atendiendo al calculado por el WEF (2013), se puede comprobar (ver Tabla 4) que existen tanto semejanzas como divergencias. Por ejemplo, mientras que Alemania, Reino Unido, Francia y España ocupan posiciones de privilegio según ambos modelos y los peores puestos del ranking están ocupados, también para ambos modelos, por Rumanía, Letonia y Lituania, estas similitudes no se producen para otros países. Así, mientras que Italia ocupa la segunda posición y Bulgaria la décima según nuestro modelo, el WEF (2013) les coloca, respectivamente, en unas alejadas decimoquinta y vigesimosexta posición. En sentido contrario, mientras que Austria y Suecia se colocan en nuestro modelo en una novena y decimosexta plaza, en el del WEF (2013) se sitúan en segunda posición y en sexta posición.

A continuación, hemos considerado interesante realizar una triple comparación considerando los dos modelos estudiados -el MCOCDT y el WEF (2013)- y el ranking por entradas de turistas. Como puede apreciarse en la Tabla 4, los cinco países de la Unión Europea que logran atraer a un mayor número de turistas internacionales son los mismos que los que ocupan las cinco primeras posiciones según nuestro modelo, aunque cambie el orden, hecho que no ocurre para el WEF (2013) pues Italia no sólo no se encuentra 
entre los cinco primeros destinos de la Unión Europea, sino que se va a la decimoquinta posición. Sin embargo, mientras que según nuestro ranking Luxemburgo ocupa la sexta posición, por volumen de entradas se va a la vigesimoctava posición; o Polonia, que según el modelo propuesto se situaría en decimonovena posición, y realmente es el octavo país de la Unión Europea por entradas de turistas.

Tabla 4

RANKING POR COMPETITIVIDAD

DE LOS PAÍSES DE LA UE SEGÚN EL MCOCDT Y WEF (2013)

Y ENTRADAS DE TURISTAS

\begin{tabular}{|l|c|c|c|c|c|c|}
\hline \multicolumn{1}{|c|}{ PAIS } & MCOCDT & POSICIÓN & WEF (2013) & POSICIÓN & $\begin{array}{c}\text { ENTRADAS } \\
\text { TURISTAS (*) }\end{array}$ & POSICIÓN \\
\hline ALEMANIA & 631,589 & $1^{\circ}$ & 5,39 & $1^{\circ}$ & 31.545 & $4^{\circ}$ \\
\hline ITALIA & 626,846 & $2^{\circ}$ & 4,9 & $15^{\circ}$ & 47.704 & $3^{\circ}$ \\
\hline R.UNIDO & 603,351 & $3^{\circ}$ & 5,38 & $3^{\circ}$ & 31.169 & $5^{\circ}$ \\
\hline FRANCIA & 578,918 & $4^{\circ}$ & 5,31 & $5^{\circ}$ & $83013(1)$ & $1^{\circ}$ \\
\hline ESPAÑA & 550,431 & $5^{\circ}$ & 5,38 & $4^{\circ}$ & 60.661 & $2^{\circ}$ \\
\hline LUXEMBURGO & 543,005 & $6^{\circ}$ & 4,93 & $13^{\circ}$ & $905(1)$ & $28^{\circ}$ \\
\hline BELGICA & 487,519 & $7^{\circ}$ & 5,04 & $9^{\circ}$ & 7.642 & $16^{\circ}$ \\
\hline DINAMARCA & 484,853 & $8^{\circ}$ & 4,98 & $12^{\circ}$ & $8068(1)$ & $15^{\circ}$ \\
\hline AUSTRIA & 484,252 & $9^{\circ}$ & 5,39 & $2^{\circ}$ & 24.813 & $6^{\circ}$ \\
\hline BULGARIA & 477,704 & $10^{\circ}$ & 4,38 & $26^{\circ}$ & 6.897 & $18^{\circ}$ \\
\hline HOLANDA & 466,542 & $11^{\circ}$ & 5,14 & $7^{\circ}$ & 12.797 & $9^{\circ}$ \\
\hline GRECIA & 455,396 & $12^{\circ}$ & 4,75 & $19^{\circ}$ & 17.923 & $7^{\circ}$ \\
\hline PORTUGAL & 448,544 & $13^{\circ}$ & 5,01 & $10^{\circ}$ & 8.324 & $14^{\circ}$ \\
\hline CROACIA & 444,179 & $14^{\circ}$ & 4,59 & $20^{\circ}$ & 10.955 & $10^{\circ}$ \\
\hline REP.CHECA & 441,172 & $15^{\circ}$ & 4,78 & $18^{\circ}$ & 9.004 & $13^{\circ}$ \\
\hline SUECIA & 431,636 & $16^{\circ}$ & 5,24 & $6^{\circ}$ & $10914(1)$ & $11^{\circ}$ \\
\hline MALTA & 430,337 & $17^{\circ}$ & 4,92 & $14^{\circ}$ & 1.582 & $26^{\circ}$ \\
\hline IRLANDA & 421,258 & $18^{\circ}$ & 5,01 & $11^{\circ}$ & $7550(1)$ & $17^{\circ}$ \\
\hline POLONIA & 415,925 & $19^{\circ}$ & 4,47 & $23^{\circ}$ & 15.845 & $8^{\circ}$ \\
\hline FINLANDIA & 415,245 & $20^{\circ}$ & 5,1 & $8^{\circ}$ & $4226(1)$ & $19^{\circ}$ \\
\hline CHIPRE & 414,769 & $21^{\circ}$ & 4,84 & $16^{\circ}$ & 2.405 & $21^{\circ}$ \\
\hline ESLOVAQUIA & 410,894 & $22^{\circ}$ & 4,32 & $27^{\circ}$ & 1.653 & $25^{\circ}$ \\
\hline HUNGRIA & 407,185 & $23^{\circ}$ & 4,51 & $22^{\circ}$ & 10.675 & $12^{\circ}$ \\
\hline ESTONIA & 396,258 & $24^{\circ}$ & 4,82 & $17^{\circ}$ & 2.868 & $20^{\circ}$ \\
\hline ESLOVENIA & 392,153 & $25^{\circ}$ & 4,58 & $21^{\circ}$ & 2.259 & $22^{\circ}$ \\
\hline LETONIA & 390,965 & $26^{\circ}$ & 4,43 & $24^{\circ}$ & 1.536 & $27^{\circ}$ \\
\hline LITUANIA & 388,752 & $27^{\circ}$ & 4,39 & $25^{\circ}$ & $1900(1)$ & $23^{\circ}$ \\
\hline RUMANIA & 381,019 & $28^{\circ}$ & 4,04 & $28^{\circ}$ & 1.715 & $24^{\circ}$ \\
\hline
\end{tabular}

(*) Previsión de entradas de turistas en 2013 en miles.

(1) Entradas de turistas en 2012 en miles.

Fuente: Rodríguez-Antón, dir. (2015). 
En cuanto a la dispersión relativa de los datos, las entradas de turistas presentan un coeficiente de variación de 1,268, muy superior al registrado para los dos indicadores de competitividad calculados a través del MOCCDT -0,157- y a través del WEF (2013) $-0,075-$.

Para finalizar este análisis, hemos calculado la correlación existente entre las entradas de turistas internacionales y las competitividades de estos destinos turísticos medidas a través de nuestro modelo y del WEF (2013). Pues bien, el MCOCDT se ajusta en mayor medida a la capacidad de atracción turística de estos destinos que el propuesto por el WEF (2013) dado que el coeficiente de correlación existente en el primer caso es de 0,732, mientras que en este último se queda en un $0,546$.

\section{CONCLUSIONES, LIMITACIONES Y FUTURAS LÍNEAS DE INVESTIGACIÓN}

Aunque el sector turismo es, desde hace décadas, el que más contribuye a la generación del Producto Interior Bruto de nuestro país, y aunque seguimos ocupando posiciones líderes en el mundo tanto por la entrada de turistas como por los ingresos generados por el turismo o como, muy recientemente, por el nivel de competitividad, no podemos dejar de ser conscientes de que una serie de factores, tanto exógenos como endógenos, pueden llegar a provocar que dejemos de ocupar estos puestos de privilegio, con las repercusiones que ello traería tanto a las empresas del sector como al resto de la economía española.

Para que España siga siendo un destino turístico de referencia, va a ser necesario que sigamos siendo competitivos a nivel internacional. En este contexto se ha desarrollado el presente estudio que ha pretendido proponer un nuevo Modelo cuantitativo objetivo de competitividad de los destinos turísticos (MCOCDT), medir el grado de competitividad turística de los países de la Unión Europea utilizando este Modelo y proponer medidas de actuación, tanto a nivel de apoyo de las Administraciones Públicas implicadas, como de acción por parte de las empresas y grupos empresariales turísticos, que eleven el grado de competitividad turística de nuestro país.

Para ello, partiendo de los catorce pilares que configuran el Modelo del WEF (2013), se han buscado indicadores cuantitativos objetivos de las variables que lo integran en aras a sustituir los indicadores de tipo blando, basados en opiniones de expertos, muy abundantes en dicho modelo. En definitiva, lo que se pretendía era que todos los indicadores del nuevo modelo fuesen de tipo duro, por tanto, procedentes de fuentes estadísticas o de bases de datos. En concreto, de las 79 variables que configuraban el modelo de referencia, 48 de tipo duro y 31 de tipo blando, se pasó a 62 variables, todas ellas cuantitativas objetivas, medidas a través de indicadores de tipo duro.

Tras el estudio empírico realizado, ha resultado que los pilares mejor valorados por los expertos como integrantes del modelo de competitividad turística fueron las Infraestructuras del transporte aéreo, seguido por las Infraestructuras turísticas, la Seguridad y protección, los Recursos culturales, la Salud e higiene y los Recursos naturales.

El modelo lineal cuantitativo objetivo de competitividad turística creado se ha aplicado a los 28 países de la Unión Europea (UE), resultando que el país que ha logrado obtener 
el mayor índice de competitividad de este espacio es Alemania, seguido por Italia, Reino Unido, Francia y España. Nuestro país se sitúa en primera posición de la Unión Europea atendiendo a la Sostenibilidad ambiental, en segunda posición por Recursos culturales y en tercera por la Prioridad del turismo y del sector de viajes para el destino y por las Infraestructuras turísticas.

Si atendemos a la posición que ocupan los veintiocho países de la UE según ambos rankings, vemos que existen tanto semejanzas como divergencias. Por ejemplo, mientras que Italia ocupa la segunda posición y Bulgaria la décima según nuestro modelo, el WEF (2013) les coloca, respectivamente, en unas alejadas decimoquinta y vigesimosexta posición. En sentido contrario, mientras que Austria y Suecia se colocan en nuestro modelo en una novena y decimosexta plaza, en el del WEF (2013) se sitúa en segunda posición y en sexta posición.

Si se realiza una triple comparación entre el nuevo modelo propuesto, el de referencia y el volumen de entradas de turistas internacionales a cada destino turístico, también surgen semejanzas y discrepancias. Por ejemplo, los cinco países de la Unión Europea que logran atraer a un mayor número de turistas internacionales son los mismos que los que ocupan las cinco primeras posiciones según nuestro modelo, aunque cambie el orden, hecho que no ocurre para el WEF (2013) pues Italia no sólo no se encuentra entre los cinco primeros destinos de la Unión Europea, sino que se va a la decimoquinta posición según este modelo.

En todo caso, se ha comprobado que la correlación existente entre las entradas de turistas internacionales y las competitividades de estos destinos turísticos calculadas a través del MCOCDT -0,732- es considerablemente superior a la existente entre dichas entradas y la competitividad medida a través del modelo del WEF (2013) -0,546-, lo cual indicaría que nuestro modelo se ajusta en mayor medida a la capacidad de atracción turística de estos destinos que el modelo de referencia.

A partir de la realización del presente trabajo, se puede inferir que nuestras Administraciones Públicas y, en segundo término, las empresas privadas, deben intentar mejorar las infraestructuras de telecomunicaciones, la apertura de nuestro país al turismo, la salud e higiene existente en España y el grado de cualificación de nuestros recursos humanos. En concreto, dentro de las infraestructuras de telecomunicaciones debería potenciarse, aún más, el uso de Internet, debería ampliarse el número de líneas de teléfono tanto fijo como móvil y debería ampliarse el uso de Internet de banda ancha.

Por lo que respecta a la apertura de nuestro país al turismo, es necesario elevar los ingresos por turismo en relación con el PIB de nuestro país. Aunque nuestro país se sitúa en los primeros puestos del mundo por ingresos procedentes del turismo, es necesario seguir incrementándolos realizando una decidida apuesta por aquellos productos y segmentos de turistas que se orienten, en mayor medida por un turismo de calidad que por uno de precios bajos. Eso no significa que tengamos que abandonar el turismo de sol y playa que logra un elevado volumen de entradas de turistas y que permite unas elevadas tasas de empleo, sino que debemos orientarnos, también, a la oferta de productos turísticos de alto valor añadido y generadores de ingresos por turista más elevados.

En cuanto al tema de salud e higiene, nuestro país, a pesar de tener un muy buen sistema sanitario, debe incrementar el número de camas de hospitales por cada 1.000 
habitantes. Por último en cuanto al grado de cualificación de nuestros recursos humanos, nuestro país, a pesar de contar con un amplio número de universidades, posee menos que los países de nuestro entorno europeo. Igualmente, España cuenta con un porcentaje de personas con edades comprendidas entre los 25 y los 64 que tienen, por lo menos, un grado en secundaria, inferior al de estos países. Por último y, especialmente, uno de los más graves problemas que afectan estructuralmente a nuestro país desde hace décadas sigue siendo la elevada tasa de desempleo de nuestra población.

La realización del presente trabajo se ha enfrentado a un conjunto de limitaciones. En primer lugar, tal y como se recoge en la literatura, lograr encontrar datos cuantitativos objetivos sobre todos los aspectos que determinan la competitividad de los destinos turísticos no es sencillo. En algunos casos esta dificultad ha procedido de la propia inexistencia de datos estadísticos sobre algunas variables como, por ejemplo, la red de transporte aéreo de la que dispone el destino, el uso de las TICs para realizar transacciones de tipo B2B o el impacto del SIDA en los negocios. El WEF (2013) solventa esta dificultad realizando una encuesta, la Executive Opinion Survey, a través de la que consigue la opinión que un conjunto de expertos tienen sobre estas variables, pero se descartó utilizar esta metodología basada en datos blandos. En otros casos, la dificultad ha procedido de la imposibilidad de acceder a bases de datos existentes, pero que requerían que la institución a la que pertenecían los investigadores estuviese suscrita a las mismas. Este es el caso de la International Air Transportation Association. Otra limitación se ha producido al intentar lograr todos los datos para el mismo año, en este caso 2013. Incluso las publicaciones de la propia Organización Mundial del Turismo, a fecha de la finalización del presente estudio, no tenía publicadas, como definitivas, las cifras de entradas de turistas en unos cuantos países a los que se aplicó el modelo. Además, no todas las fuentes estadísticas consultadas ofrecían todos los datos relativos a los indicadores seleccionados para todos los países que integran la UE.

Asimismo, la inexistencia de fuentes estadísticas homogéneas a nivel mundial para todos los indicadores utilizados en el modelo ha dificultado la selección de un número más amplio de países a considerar en el estudio. Aunque la UE es un buen marco de estudio, pues buena parte de los destinos turísticos mundiales más demandados se encuentran en dicho espacio, no cabe duda que importantes destinos turísticos como Estados Unidos, China, Turquía, Rusia o Tailandia han quedado fuera del estudio por no pertenecer al mismo.

Igualmente, por lo que respecta a la representatividad de la muestra seleccionada para el cálculo de los pesos relativos de los pilares y de las variables, al ser una muestra de conveniencia, puede ser cuestionada por no poder calcularse su nivel de significatividad, nivel de confianza y otras probabilidades asociadas, además de no existir un universo muestral de referencia.

Para finalizar, este trabajo abre futuras líneas de investigación al suponer un primer paso para llegar a determinar un modelo cuantitativo objetivo de competitividad de los destinos turísticos basado, única y exclusivamente, en datos duros y supone el comienzo de futuras líneas de investigación que van a partir del mismo. En concreto, se va continuar calculando, año a año, la competitividad de los destinos turísticos de la Unión Europea a partir del nuevo modelo creado. Ello nos permitirá evaluar, de manera dinámica, la evo- 
lución que estos destinos están experimentando con el tiempo, llegando a diagnosticar los motivos por los que están ganando o perdiendo competitividad.

Igualmente, sería interesante ampliar la aplicación del MCOCDT a otras zonas o espacios geográficos, geopolíticos o geoeconómicos -por ejemplo, tal y como estructura el mundo la OMT, Europa, América, Asia-Pacífico, Oriente Medio y Norte de África, y África Subsahariana- hasta llegar, en la medida de lo posible, a aplicar dicho modelo a escala mundial. Por último, se va a continuar intensificando en la búsqueda de indicadores cuantitativos objetivos que puedan medir, con mayor precisión, la competitividad turística.

\section{BIBLIOGRAFÍA}

CAMPBELL, D.T. (1970): «Natural selection as an epistemological model». In Raoul Naroll and Ronald Cohen (Eds.) A handbook of method in cultural anthropology, New York, National History Press, pp. 51-85.

CAMPBELL, D.T. y FISKE, D.W. (1959): «Convergent and discriminant validation by the multitrait-multimethod matrix». Psychological Bulletin, $\mathrm{n}^{\circ}$ 56, pp. 81-105.

CAMPBELL, D.T. y OVERMAN, E.S. (1988): Methodology and epistemology for social science: Selected papers. University of Chicago Press.

CLAVER, E., MOLINA, J. y PEREIRA, J. (2007): «Competitiveness in mass tourism». Annals of Tourism Research, vol. 34 (3), pp. 727-745.

COOK, T.D. y REICHARDT, CH.S. (1986): Métodos Cualitativos y cuantitativos en investigación evaluativa. Madrid, Ediciones Morata.

COSTA, C. (2001): «Novas estratégias para o Turismo». Comunicaçao no Seminário da $A E P$. Europarque. Julio.

CRACOLICI, M. y NIJKAMP, P. (2009): «The attractiveness and competitiveness of tourist destinations: a study of southern italian regions». Tourism Management, vol. 30 (3), pp. 336-344.

CROUCH, G.I. y RITCHIE, J. (1999): «Tourism, competitiveness and societal prosperity». Journal of Business Research, vol. 44 (3), pp. 137-152.

DE KEYSER, R. y VANHOVE, N. (1994): «The competitive situation of tourism in the Caribbean area-Methodological approach». Revue de Tourisme, vol. 3, pp. 19-22.

D'HAUTESERRE, A.M. (2000): «Lessons in managed destination competitiveness: the case of Foxwoods Casino Resort». Tourism Management, vol. 21, pp. 23-32.

DWYER, L. y KIM, C. (2003): «Destination competitiveness: Determinants and indicators», Current Issues in Tourism, 6 (5), pp. 369-414.

DWYER, L., KNEZEVIC, L., MIHALIC, T. y KOMAN, M. (2014): «Integrated destination competitiveness model: testing its validity and data accessibility». Tourism Analysis, vol. 19 (1), pp. 1-17.

GARAU, J. (2006): «Propuesta de dos índices para la medición de la competitividad de los destinos de sol y playa del Mediterráneo: avance de resultados desde el punto de vista de la demanda». XV Simposio Internacional de turismo y ocio. ESADE- Fira de Barcelona. 3 de mayo de 2006.

GOMEZELJ, D.O. y MIHALIC, T. (2008): «Destination competitiveness. Applying different models, the case of Slovenia». Tourism Management, vol. 29 (2), pp. 294-307. 
GONZÁLEZ, R. y MENDIETA, M. (2009): «Reflexiones sobre la conceptualización de la competitividad de destinos turísticos». Cuadernos de Turismo, $\mathrm{n}^{\circ} 23$, pp. 111-128.

HASSAN, S.S. (2000): «Determinants of market competitiveness in an environmentally sustainable tourism industry». Journal of Travel Research, vol. 38 (3), pp. 239-245.

HERNÁNDEZ, R. (1998): Metodología de la Investigación. Mc Graw Hill.

HONG, W.CH. (2008): Competitiveness in the Tourism Sector: a comprehensive approach from Economic and Management Points. Heidelberg, Physica-Verlag,

HONG, W.CH. (2009): «Global competitiveness measurement for the tourism sector». Current Issues in Tourism, vol. 12 (2), pp. 105-132.

JANG, S. y FENG, R. (2006): «Temporal destination revisit intention: the effects of novelty seeking and satisfaction». Tourism Management, vol. 28 (2), pp. 580-590.

JIMÉNEZ, P. y AQUINO, F.K. (2012): «Propuesta de un modelo de competitividad de destinos turísticos». Estudios y Perspectivas en Turismo, vol. 21 (4), pp. 977-995.

KIM, C., CHOI, K., DWYER, L., FAULKNER, B., MELLOR, R. y LIVAIC, Z. (2001): Destination competitiveness: development of a model with application to Australia and the Republic of Korea. Department of Industry Science and Resources, AustraliaKorea Fundation.

KOZAC, M. y RIMMINGTON, M. (1999): «Measuring tourist destination competitiveness: conceptual considerations and empirical findings». International Journal of Hospitality Management, vol. 18, 273-283.

MAZANEC, J., WÖBER, K. y ZINS, A. (2007): «Tourism destination competitiveness: From definition to explanation?», Journal of Travel Research, vol. 46, pp. 86-95.

MONFORT, V. (1999): Competitividad y factores críticos de éxito en los destinos turísticos mediterráneos: Benidorm y Peñíscola. Tesis Doctoral. Valencia: Universidad de Valencia.

ORTA, F.J. (2005): «Retos para la competitividad de la industria turística en el Principado de Asturias». Revista de Economía, Sociedad, Turismo y Medio Ambiente, $\mathrm{n}^{\circ}$ 2, pp. 57-63.

PERLES-RIBES, J.F., RAMÓN-RODRÍGUEZ, A.B. y SEVILLA-JIMÉNEZ, M. (2014): «La cuota de mercado como indicador de competitividad en los destinos turísticos: sentido y limitaciones». Cuadernos de Turismo, no 34, pp. 265-285.

PORTER, M.E. (1985): Competitive advantage. New York, Free Press.

PORTER, M.E. (1990): «The competitive advantage of nations». Harvard Business Review, vol. 68 (2), pp. 73-93.

RICARDO, D. (1817): On the Principles of Political Economy and Taxation. London, John Murray.

RITCHIE, J. y CROUCH, G.I. (2000): «The competitive destination. A Sustainable perspective». Tourism Management, vol. 21 (1), pp. 1-7.

RITCHIE, J. y CROUCH, G.I. (2003): The competitive destination. A Sustainable perspective. Cambridge, CABI Publishing.

RODRÍGUEZ-ANTÓN, J.M. (Dir.) (2015): Análisis de la competitividad de España como destino turístico. Madrid, ACCI. 
RODRÍGUEZ-ANTÓN, J.M. y RUBIO-ANDRADA, L. (2016): «Estudio de la competitividad turística de la Unión Europea a partir del modelo cuantitativo objetivo». Esic Market, vol. 47 (2), pp. 209-232.

SAVERIADES, A. (2000): «Establishing the social tourism carrying capacity for tourist resorts of the coast of republic of Cyprus». Tourism Management, vol. 21, pp. 147-156. WEAVER, D.B. (2000): «A broad context model of destination development scenarios. Tourism Management, vol. 21 (3), pp. 217-224.

WORLD ECONOMIC FORUM (WEF) $(2007,2009,2011,2013,2015)$. The Travel \& Tourism Competitiveness Report. Switzerland. 
